LA GRANJA:

REVISTA DE

CIENCIAS DE LA VIDA

pISSN:1390-3799; eISSN:1390-8596

http://doi.org/10.17163/lgr.n33.2021.02
Artículo científico / Scientific paper

LA COMPRENSIÓN DE LA VACA LECHERA

PARA UNA PRODUCCIÓN SUSTENTABLE

\title{
PRESENCIA DE METAles PESAdOS EN LECHE CRUDA BOVINA DE MACHACHI, ECUADOR
}

\author{
PRESENCE OF HEAVY METALS IN RAW BOVINE MILK FROM MACHACHI, \\ ECUADOR
}

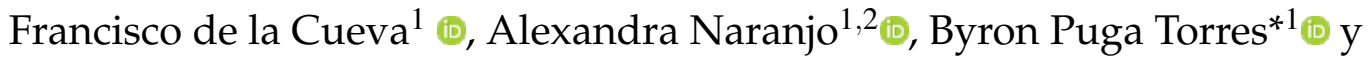 Eduardo Aragón ${ }^{1}$

\author{
${ }^{1}$ Facultad de Medicina Veterinaria y Zootecnia. Universidad Central del Ecuador, Jerónimo Leyton s/n y Gatto Sobral, Av. Uni- \\ versitaria, Quito, 170129, Ecuador. \\ 2 Trouw Nutrition Latam. \\ *Autor para correspondencia: bpuga@uce.edu.ec
}

\begin{abstract}
Resumen
La evaluación de metales pesados en la leche puede considerarse como indicador de contaminación ambiental de un lugar, por lo que el objetivo de la investigación fue determinar la presencia de plomo, mercurio y arsénico en leche cruda de Machachi, Provincia de Pichincha-Ecuador. Se recolectaron 58 muestras provenientes de 29 fincas lecheras con sistema de pastoreo extensivo y ubicadas hasta máximo un kilómetro a la redonda del Municipio de Machachi, donde existe actividad industrial y está cerca de la Panamericana Sur. Las muestras fueron analizadas mediante la técnica de espectrofotometría de absorción atómica por generador de hidruros (mercurio y arsénico) y con horno de grafito (plomo). Todas las muestras analizadas mostraron niveles de plomo, con una media de $0,208 \mathrm{mg} \mathrm{kg}^{-1}$ ( $\mathrm{rango}$ entre 0,0016 a 0,719 $\left.\mathrm{mg} \mathrm{kg}^{-1}\right)$, de las cuales el 98,28\% (57/58) contienen niveles superiores a los máximos permitidos por la NTE INEN 9 de $0,02 \mathrm{mg} \mathrm{kg}^{-1}$. También se detectó mercurio y arsénico en cuatro y dos muestras de leche, respectivamente, encontrándose en el primer caso en una media de $0,00009 \mathrm{mg} \mathrm{kg}^{-1}$ (rango entre $0,00 \mathrm{a} 0,002 \mathrm{mg} \mathrm{kg}^{-1}$ ) y en el segundo caso un promedio de $0,00003 \mathrm{mg} \mathrm{kg}^{-1}\left(0,00 \mathrm{a} 0,001 \mathrm{mg} \mathrm{kg}^{-1}\right)$, y aunque los niveles son muy bajos, los mismos son bastante preocupantes ya que son capaces de acumularse y ser potencialmente cancerígenos. Con los resultados obtenidos, se concluye que se ha detectado contaminación por plomo en el área estudiada, pero sobre todo por dos sustancias altamente tóxicas (arsénico y mercurio), para lo cual es necesario un monitoreo continuo en la leche, y sobre todo para buscar posibles fuentes de contaminación.
\end{abstract}

Palabras clave: Plomo, Arsénico, Mercurio, leche cruda, Machachi. 


\begin{abstract}
The presence of heavy metals in milk is an indicator of local environmental contamination. The objective of this investigation was to test raw milk from Machachi, Pichincha Province, Ecuador for the presence of lead, mercury, and arsenic. Fifty eight samples were collected from twenty nine dairy farms with extensive grazing systems located up to one kilometer from the Municipality of Machachi. Machachi is a site of industrial activity and is located near the Panamericana Sur highway. The samples were analyzed using atomic absorption spectrophotometry with a hydride generator (mercury and arsenic) and a graphite furnace (lead). All analyzed samples indicated the presence of lead, with an average abundance of $0.208 \mathrm{mg} \mathrm{kg}^{-1}$ (range between 0.0016 to $0.719 \mathrm{mg} \mathrm{kg}^{-1}$ ). Of these samples, $98.28 \%$ $(57 / 58)$ contained lead at levels higher than the maximum allowed by the NTE INEN 9, $0.02 \mathrm{mg} \mathrm{kg}^{-1}$. Mercury was detected in four samples with a mean abundance of $0.00009 \mathrm{mg} \mathrm{kg}^{-1}$ (range between 0.00 to $0.002 \mathrm{mg} \mathrm{kg}^{-1}$ ); and arsenic was detected in two samples with a mean abundance of $0.00003 \mathrm{mg} \mathrm{kg}^{-1}\left(0.00\right.$ to $\left.0.001 \mathrm{mg} \mathrm{kg}{ }^{-1}\right)$. Although these levels are very low, they remain worrisome because these carcinogenic elements are capable of accumulating. Based on these results, it can be concluded that lead contamination has occurred in the studied area. Furthermore, the detection of arsenic and mercury, two highly toxic substances, warrants continuous monitoring of the regional milk supply and a search for possible sources of contamination.
\end{abstract}

Keywords: Lead, Arsenic, Mercury, raw milk, Machachi.

Forma sugerida de citar: De la Cueva, F., Naranjo, A., Puga-Torres, B. y Aragón, E. (2021). Presencia de metales pesados en leche cruda bovina de Machachi, Ecuador. La Granja: Revista de Ciencias de la Vida. Vol. 33(1):2130. http://doi.org/10.17163/lgr.n33.2021.02.

IDs Orcid:

Francisco de la Cueva: http:/ / orcid.org/0000-0003-2671-0329

Alexandra Naranjo: http:/ /orcid.org/0000-0002-6442-0982

Byron Puga Torres: http:/ /orcid.org/0000-0002-4444-0054

Eduardo Aragón: http:/ / orcid.org/0000-0002-5142-0721 


\section{Introducción}

En 2018, la producción de leche ecuatoriana alcanzó 5 millones de litros por día, con más del 70\% de la producción en la región interandina. Pichincha es la mayor provincia productora de leche, representando aproximadamente el $16 \%$ del suministro total de leche en el Ecuador (INEC, 2019). El cantón Mejía ha sido conocido históricamente como el mayor productor lechero de la provincia de Pichincha y en todo el Ecuador, debido en gran parte a sus condiciones climáticas y de suelo, que son muy favorables para el desarrollo ganadero (CIL, 2015). La Norma Técnica Ecuatoriana (NTE) INEN 9 (INEN, 2012) y el Codex Alimentarius (Codex Alimentarius, 1995) establecen normas ambientales para la leche cruda y determinan el nivel máximo permitido de plomo $(\mathrm{Pb})$ en $0,02 \mathrm{mg} / \mathrm{kg}$. La norma NTE INEN 9 no indica los niveles máximos permitidos de mercurio (Hg) o arsénico (AS); sin embargo, la NTE INEN 1108, que se ocupa del agua potable, establece un límite máximo de $0,006 \mathrm{mg} / \mathrm{L}$ para $\mathrm{Hg}$ y 0,01 mg/L para As (INEN, 2011).

La presencia de metales pesados en los alimentos representa un gran problema para la salud pública, ya que estos elementos causan daños en la salud de los humanos, animales y en los cultivos agrícolas (Anastasio y col., 2006). En los seres humanos, los metales pesados interrumpen las funciones del sistema nervioso, el hígado y el riñón, al tiempo que promueven la mutagénesis y la carcinogénesis. En los animales, los metales pesados pueden causar pérdida del apetito, anemia, trastornos reproductivos, cáncer y teratogénesis. Todos estos efectos disminuyen el rendimiento de la producción a largo plazo (González-Montaña, 2009). La principal fuente de metales pesados es la contaminación ambiental resultante de la presencia de diversas actividades industriales (Zhou y col., 2019). Estas actividades contaminan el suelo (Ashraf y col., 2019), el agua y el aire; y eventualmente llegan a personas y animales a través de la cadena alimentaria. Por lo tanto, esta contaminación perjudica enormemente la salud de los consumidores (Karasakal, 2020). Actualmente, esta contaminación se mide mediante el uso de biomonitoreo, dentro del cual la vigilancia de los productos animales desempeña un papel importante (Scaramozzino y col., 2019). En el caso de la producción de productos lácteos, los metales pesados pueden contaminar el agua y los alimentos de las vacas lecheras, trasladándose eventualmente a la leche (Zhou y col., 2019). Esto constituye un serio problema de salud pública, especialmente para las poblaciones vulnerables como los niños (ChirinosPeinado y Castro-Bedriñana, 2020), porque incluso los bajos niveles de metales pesados incorporados en una dieta pueden conducir a enfermedades crónicas (Miclean y col., 2019). Así, el monitoreo de la presencia de metales pesados en la leche cruda producida localmente resulta un excelente indicador de contaminación ambiental en la región (Miclean y col., 2019).

El plomo $(\mathrm{Pb})$ se produce con frecuencia por la descomposición radioactiva del uranio y el actinio, y se puede encontrar en el suelo (Silva y col., 2010). Sin embargo, el plomo también puede introducirse en el medio ambiente a través de otras vías, como el uso de equipos que contienen plomo, fertilizantes químicos y pesticidas, o agua contaminada con plomo (Litter, Farías y Armienta, 2009). El plomo es uno de los intoxicantes más frecuentes en el ganado, especialmente en los terneros, ya que estos animales carecen de una microflora ruminal funcional (Perrin, Schiefer y Blakley, 1990). En el caso del arsénico, éste puede distribuirse en el agua, el aire y el suelo. El arsénico es muy tóxico en su forma inorgánica, y puede causar cambios en la piel e incluso cáncer (WHO, 2018). La presencia de mercurio también implica un problema debido a su capacidad para contaminar y dañar la cadena trófica. La exposición crónica a este metal puede resultar en toxicidad asintomática (Rodríguez, López-Huertas y Boza Puerta, 2010). El mercurio es el único metal volátil, por lo que se absorbe fácilmente a través de la piel y los pulmones. Su presencia en el medio ambiente se debe principalmente a la actividad humana y es comúnmente introducido por los sistemas de calefacción, la minería y otros procesos industriales (WHO, 2019).

La contaminación del aire se compone de partículas finas que pueden combinar y transportar sustancias tóxicas tales como los metales pesados. Esto permite el transporte de estos metales a través del aire y los deposita en agua de riego, suelos agrícolas y pastos (Dergham y col., 2012; Alloway, 2013; Yilmaz, Akinci y Akinci, 2009). En el contexto de las vacas lecheras, si el forraje contiene materiales pesados, esos metales tóxicos se introducen en el animal y finalmente se pasan a su leche (Miclean y col., 
2019), razón por la cual es necesario estudiar este proceso (Oliveira y col., 2017) para estimar los efectos toxicológicos (Samiee, Javad y Leili, 2019) y proteger la salud pública (Miclean y col., 2019). Hasta el momento, pocos estudios han investigado la presencia de metales pesados en la leche cruda de Ecuador, a pesar de la máxima importancia de este proceso de monitoreo para prevenir la contaminación de los alimentos (Kim y col., 2016) y la transferencia de metales pesados a los consumidores (Hashemi, 2018). El objetivo de este estudio fue cuantificar los niveles de plomo, arsénico y mercurio en la leche cruda producida en la mayor cuenca lechera del Ecuador.

\section{Materiales y métodos}

\subsection{Ubicación del área de estudio}

El estudio se realizó en la parroquia de Machachi, Cantón Mejía, Provincia de Pichincha, Ecuador. Se recolectaron 58 muestras de leche cruda al azar de 29 unidades de producción lechera (UPA), con extensos sistemas de pastoreo ubicados a un kilómetro de la ciudad de Machachi, que es un centro de actividad industrial especializado en la producción de acero, y ubicado cerca de la carretera Panamericana Sur. Las UPA están situadas cerca de -0,510110, 78,567123 , a una altitud de aproximadamente 2900 metros sobre el nivel del mar (GeoDatos, 2019). La región experimenta temperaturas de 12 a $20^{\circ} \mathrm{C}$, y generalmente se caracteriza por tener un clima fríotemplado. La ubicación geográfica se obtuvo utilizando georreferenciación basada en el sistema de posicionamiento global (GPS), como se muestra en la Figura 1.

\subsection{Muestreo}

Se tomaron aproximadamente $250 \mathrm{ml}$ de leche cruda directamente de los tanques de refrigeración, cubos o contenedores de recolección en cada UPA. Esta operación de muestreo se repitió de nuevo en el plazo de ocho días y se llevó a cabo de conformidad con NTE INEN-ISO 707: “Leche y productos lácteos. Instrucciones para el muestreo" (INEN, 2014b). Las muestras se transportaron en envases que contenían refrigerantes congelados en gel entre 2 y $5 \mathrm{oC}$. A su llegada al Laboratorio del Departamento de Petróleo, Energía y contaminación (DPEC) de la Facultad de Ingeniería Química de la Universidad Central del Ecuador, las muestras se almacenaron a $-20^{\circ} \mathrm{C}$ hasta el momento del análisis.

\subsection{Procedimiento analítico}

Para la determinación de los niveles de $\mathrm{Hg}$ y AS, se utilizó una técnica de espectrofotometría de absorción atómica con generador de hidruros (Rocha, 2011). La determinación de los niveles de $\mathrm{Pb}$ se realizó utilizando el mismo método de espectrofotometría de absorción atómica, pero con un horno de grafito. Las operaciones técnicas se realizaron de acuerdo con la especificación Técnica Ecuatoriana INEN-ISO/TS 6733 (INEN, 2014a).

Se utilizó un espectrofotómetro UNICAM Solar modelo 9626, y lámparas de cátodo para cada elemento analizado, con una vaporización realizada con una llama de aire-acetileno. De igual forma, se utilizó agua desionizada para todos los procesos analíticos. Las curvas de calibración se crearon utilizando estándares certificados, y las soluciones en blanco se prepararon y trataron de la misma forma que las muestras. El límite de detección (LD) y el límite de cuantificación (LC) para $\mathrm{Pb}$ fue de $0,1 \mathrm{mg} / \mathrm{L}$ y $0,8 \mathrm{mg} / \mathrm{L}$, respectivamente. Para As, el LD fue de $0,39 \mu \mathrm{g} / \mathrm{L}$ y el LC fue de 1,19 $\mu \mathrm{g} / \mathrm{L}$. El LD para el mercurio fue de $0,14 \mu \mathrm{g} / \mathrm{L}$ y el LC fue de $0,42 \mu \mathrm{g} / \mathrm{L}$. Todas las muestras se analizaron por triplicado. Las curvas de calibración respectivas se prepararon utilizando las siguientes concentraciones: $\mathrm{Pb}=0,0 ; 0,5$; 1,$5 ; 3,0$ y $5,0 \mathrm{mg} / \mathrm{L} ; \mathrm{Hg}=1,0 ; 2,5 ; 5,0 ; 10,0 ; 25,0$ y 50,0 $\mu \mathrm{g} / \mathrm{L} ; \mathrm{As}=0 ; 1 ; 2 ; 5, \mathrm{y} 10 \mu \mathrm{g} / \mathrm{L}$.

\subsection{Análisis estadístico}

Los resultados se expresan como la media, la concentración mínima y máxima observada de $\mathrm{Pb}, \mathrm{As}$ o Hg. Se realizó una prueba de Shapiro-Wilk para verificar la normalidad de los datos. Los resultados de estas pruebas indicaron que los datos no se distribuían normalmente $(p \leq 0,05)$. Por esta razón, se utilizaron pruebas estadísticas no paramétricas (Celis De La Rosa y Labrada, 2014). Se utilizó una prueba de Kruskal-Wallis para comparar los niveles de metales pesados en la leche cruda entre los proveedores. De igual forma, se llevó a cabo una prueba de Wilconox para comparar los datos de la primera y la segunda muestra tomadas de un proveedor determinado. El software estadístico de código abierto RStudio versión 1.2.5019 [RStudio Inc. Boston, MA, 
EE.UU.] se utilizó para realizar todas las pruebas estadísticas. Para todos los análisis se determinó un

umbral de significación de $p=0,05$.

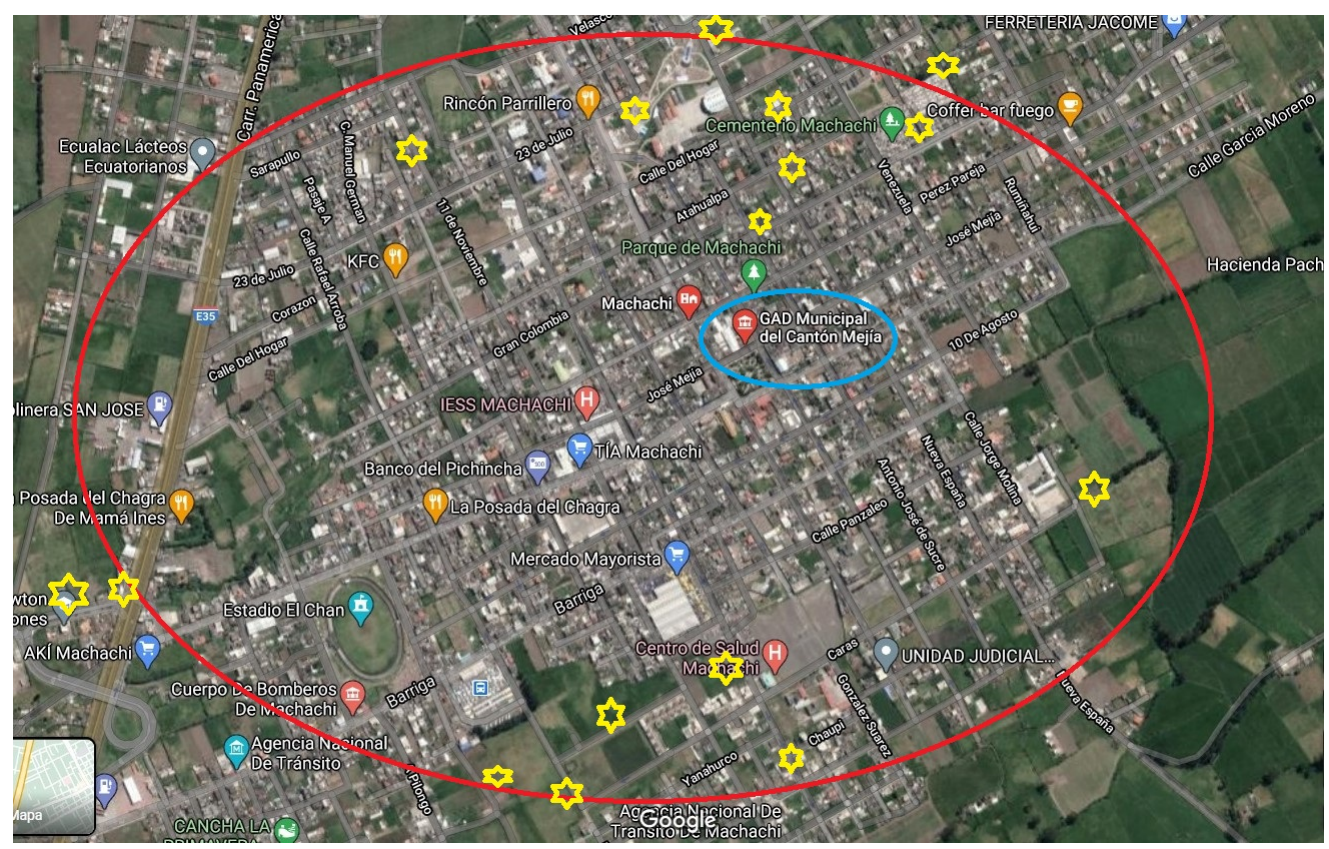

Figura 1. Unidades de producción lechera (UPA) muestreadas en la parroquia de Machachi.

\section{Resultados}

Las mínimas, medias y máximas observadas mostraron un gran número de $\mathrm{Pb}, \mathrm{Hg}$ y As en la leche cruda muestreada de Macachi, como se presenta en la Tabla 1 y la Figura 2. En el caso de Pb, el $100 \%$ $(58 / 58)$ de las muestras de leche cruda tenían niveles de plomo por encima del límite de detección de este metal pesado. El 98,28\% (57/58) contenía plomo a niveles superiores a $0,02 \mathrm{mg} / \mathrm{kg}$, que es el límite máximo establecido por NTE INEN 9 y el Codex Alimentarius. El valor medio del plomo observado en la leche cruda de la muestra fue de $0,208 \mathrm{mg} / \mathrm{kg}$, con un valor mínimo de $0,0016 \mathrm{mg} / \mathrm{kg}$ (encontrado en la primera muestra) y un valor máximo de 0,719 $\mathrm{mg} / \mathrm{kg}$ (en la segunda muestra). No se observaron diferencias significativas $(p \geq 0,05)$ al comparar la leche cruda de los 29 proveedores incluidos en la muestra, pero sí se evidenciaron diferencias significativas $(p \leq 0,05)$ entre la leche cruda de la primera muestra y la leche cruda de la segunda (Tabla 1).

En cuanto a $\mathrm{Hg}$, el 6,9\% (4/58) de las muestras de leche cruda contenían niveles detectables. El ni- vel medio de mercurio fue de $0,00009 \mathrm{mg} / \mathrm{kg}$, con un valor mínimo de $0,00 \mathrm{mg} / \mathrm{kg}$, y un valor máximo de $0,002 \mathrm{mg} / \mathrm{kg}$ (encontrado en el primer muestreo). No se detectaron diferencias significativas en relación a la presencia de mercurio entre proveedores o entre muestreos (Tabla 1). El 3,44\% (2/58) de las muestras de leche cruda analizadas contenían niveles detectables de As, con un valor medio de $0,00003 \mathrm{mg} / \mathrm{kg}$, un valor mínimo de $0,00 \mathrm{mg} / \mathrm{kg}$ y un valor máximo de $0,001 \mathrm{mg} / \mathrm{kg}$. No se detectaron diferencias significativas en la presencia de mercurio entre proveedores o entre muestreos (Tabla 1).

\section{Discusión}

Todas las muestras de leche cruda indican la presencia de $\mathrm{Pb}$, y el 98,28\% de ellas tenían niveles de plomo superiores a $0,02 \mathrm{mg} / \mathrm{kg}$, que es el máximo permitido por NTE INEN 9 y el Codex Alimentarius. Sin embargo, resulta preocupante la presencia de As y $\mathrm{Hg}$ en la leche. Aunque la gran mayoría de las muestras no contenían ninguno de estos metales pesados, el hecho de que se detectaran es alarman- 
te ya que incluso cantidades mínimas resultan tóxicas para la salud del ser humano. El mercurio, que puede ocurrir naturalmente (Bernhoft, 2012), ha sido liberado al medio ambiente durante siglos como resultado de actividades antropogénicas. Se ha demostrado que el mercurio tiene efectos nocivos en diversos tejidos y órganos del cuerpo humano (Rocha y col., 2012), y también posee potencial genotóxico. Como resultado de esto, el mercurio podría estar involucrado en la carcinogénesis, (Yang y col., 2020).

Tabla 1. Mínimas, medias y máximas de $\mathrm{Pb}, \mathrm{Hg}$ y As en la leche cruda muestreada.

\begin{tabular}{cccc|ccc|ccc}
\hline Valor & \multicolumn{3}{c|}{ Plomo $(\mathbf{m g} / \mathbf{k g})$} & \multicolumn{3}{c|}{ Mercurio $(\mathbf{m g} / \mathbf{k g})$} & \multicolumn{3}{c}{ Arsénico $(\mathbf{m g} / \mathbf{k g})$} \\
\cline { 2 - 10 } & $\begin{array}{c}\text { Primer } \\
\text { muestreo }\end{array}$ & $\begin{array}{c}\text { Segundo } \\
\text { muestreo }\end{array}$ & \multirow{2}{*}{ Total } & $\begin{array}{c}\text { Primer } \\
\text { muestreo }\end{array}$ & $\begin{array}{c}\text { Segundo } \\
\text { muestreo }\end{array}$ & Total & $\begin{array}{c}\text { Primer } \\
\text { muestreo }\end{array}$ & $\begin{array}{c}\text { Segundo } \\
\text { muestreo }\end{array}$ & Total \\
\hline Mínimo & 0,16 & 0,037 & 0,016 & 0 & 0 & 0 & 0 & 0 & 0 \\
Promedio & 0,07631 & 0,339 & 0,20764 & 0,0001 & $3 \mathrm{E}-05$ & $9 \mathrm{E}-05$ & 0 & 0,0001 & $3 \mathrm{E}-05$ \\
Máximo & 0,146 & 0,719 & 0,719 & 0,002 & 0,001 & 0,002 & 0 & 0,001 & 0,001 \\
Valor-P & $1,302 \mathrm{e}-06 *$ & $0,979 *$ & $0,3045^{*}$ & $0,052 * *$ & $0,1609 *$ & $0,492 * *$ & $0,1609 *$ & $0,492^{* *}$ \\
\hline
\end{tabular}

${ }^{*}$ Prueba de Kruskal Wallis entre los 29 proveedores. $\quad{ }^{* *}$ Prueba de Wilconox entre las muestras

Figura 2. Diagrama de caja de $\mathrm{Pb}, \mathrm{Hg}$, y As en la leche cruda analizada.

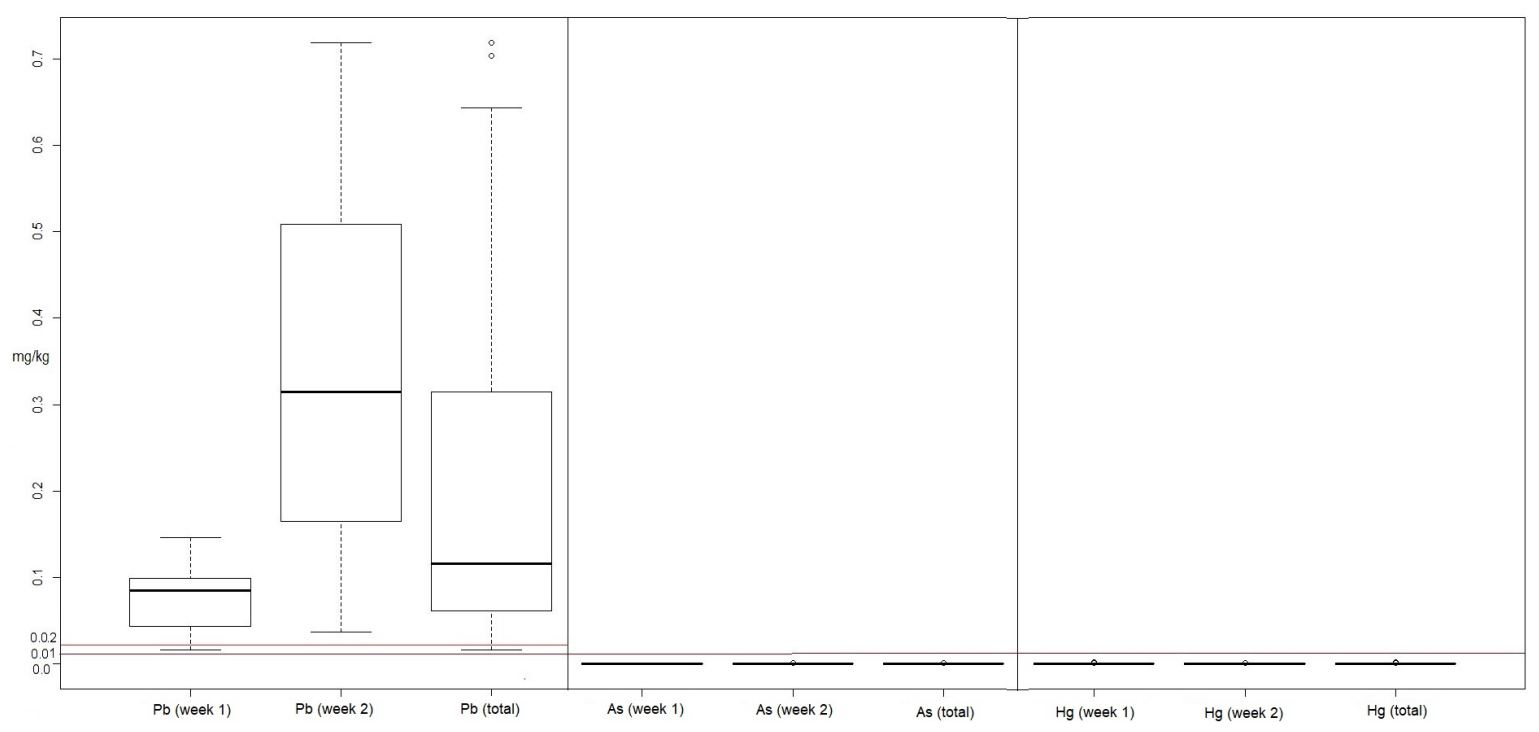

En los seres humanos, la exposición al arsénico aumenta el riesgo de cáncer de pulmón, piel y vejiga, especialmente cuando dicha exposición es crónica (Khairul y col., 2017). El arsénico también está relacionado con muchas otras afecciones patológicas conocidas como arsenicosis (Bjørklund y col., 2018). La presencia de metales pesados en la leche cruda podría deberse a que las unidades de producción lecheras se encuentran en un área con gran actividad industrial, cerca de una carretera importante (González-Montaña y col., 2019). Se sabe que los niveles de metales pesados son más altos en esas zonas (Zhou y col., 2019), y de hecho se ha reportado una acumulación de metales pesados en alfalfa en regiones industrialmente activas (Rezaeian, Kiaei y Zadeh, 2020). La contaminación puede introducirse a través de varias vías, incluyendo agua potable, pastos y/o el suelo. La determinación de la fuente de contaminación por metales pesados es fundamental para su control; de igual forma, otros estudios han reportado contaminación tanto en el forraje $(\mathrm{Pb})$ como en el agua potable (As y Hg) con- 
sumida por las vacas lecheras (Zhou y col., 2017; Zhou y col., 2019).

En Ecuador, pocos estudios han investigado la presencia de metales pesados en la leche. En un estudio que utilizó 20 muestras de leche cruda del cantón de Arenillas, provincia El Oro (Ayala y Romero, 2013), se detectaron $\mathrm{Hg}$ y As (media de $0,01035 \mathrm{mg} / \mathrm{kg}$ ) en el $100 \%$ de las muestras. Si bien esto no coincide con los resultados presentados, tal vez se pueda explicar debido al hecho de que Arenillas es un sector minero a diferencia de Machachi. Con respecto a la contaminación por $\mathrm{Pb}$, los datos en El Oro mostraron una media de $0,011 \mathrm{mg} / \mathrm{kg}$ (rango de 0,006 a 0,018 mg/kg). Este nivel de contaminación por plomo es menor que el reportado en esta investigación. Esto podría deberse a la presencia de plomo en el agua del río que abastece los canales de riego utilizados por los rebaños. Además, un estudio en la ciudad de Guayaquil encontró altos niveles de contaminación por $\mathrm{Pb}$ en la leche en polvo $(5.450 \pm 2.474 \mathrm{mg} / \mathrm{kg})$, pero no se detectó $\mathrm{Pb}$ en las leches pasteurizadas y ultrapasteurizadas del mismo estudio (Pernía et al., 2015).

La variabilidad de estos datos es común en todo el mundo. Un metaanálisis de 72 investigaciones de 37 países demostró que los niveles de metales pesados varían mucho. Algunas de las concentraciones más altas de $\mathrm{Pb}$ se observaron en Brasil, Croacia, Egipto, México, Nigeria, Palestina, Serbia y Turquía. Asimismo, se observaron niveles más altos de As y $\mathrm{Pb}$ en la leche producida con métodos tradicionales (como el empleado en Machachi) en comparación con la leche producida con sistemas orgánicos. En el caso del $\mathrm{Hg}$, todos los niveles se observaron por debajo del umbral mínimo de riesgo, independientemente del sistema de producción (Zwierzchowski y Ametaj, 2018). Si se comparan los resultados de este estudio con otras investigaciones llevadas a cabo en América Latina, la variabilidad sigue siendo significativa, incluso dentro del mismo país. En el caso de Brasil, la contaminación por Pb excedió los límites establecidos tanto en la leche cruda como en los derivados de la leche (Silva y col., 2010; Oliveira y col., 2017; Vasconcelos y col., 2019; Gomes y col., 2013). En Puebla, México, el análisis de muestras de leche cruda reveló la presencia de $\mathrm{Pb}$ a una abundancia media de $0,03 \mathrm{mg} / \mathrm{kg}$, y a una abundancia media de $0,12 \mathrm{mg} / \mathrm{kg}$. En ambos casos, la contaminación por metales pesados superó el máximo permitido por el Codex Alimentarius, pero estuvo por debajo del límite establecido por la ley mexicana. Las muestras de productos lácteos analizados en el mismo estudio también contenían $\mathrm{Pb}$ y As. Por lo tanto, tanto la leche cruda como los productos lácteos de este estudio pueden representar un riesgo para la salud de los consumidores (CastroGonzález y col., 2018a).

En otro estudio realizado con 160 muestras de leche cruda de Puebla y Tlaxcala, México, los niveles de $\mathrm{Pb}$ oscilaron entre $0,039 \pm 0,02$ y $0,059 \pm$ $0,05 \mathrm{mg} / \mathrm{kg}$, mientras que los niveles de As oscilaron entre 0,029 y 0,039 mg/kg. En esta región, el suelo recibe aguas residuales industriales, y los forrajes están compuestos principalmente de alfalfa. Estos niveles de $\mathrm{Pb}$ exceden los límites europeos y de la FAO, pero tanto la abundancia de $\mathrm{Pb}$ como la de As cumplen con las regulaciones mexicanas $(\mathrm{Pb}$ $0,1 \mathrm{mg} / \mathrm{kg}$ y $0,2 \mathrm{mg} / \mathrm{kg}$ para As). Es posible que se observen estas cantidades debido a que la alfalfa puede facilitar la bioacumulación de los metales pesados, permitiendo en última instancia su transferencia a la leche (Castro-González y col., 2018b). En un reciente estudio peruano se determinó que los metales pesados pueden transferirse de la sangre a la leche de la vaca, siendo los valores de $\mathrm{Pb}$ en la leche un $54 \%$ más altos que los niveles en la sangre. Los niveles de $\mathrm{Pb}$ observados en este estudio fueron 29 veces superiores a los permitidos por el Codex Alimentarius, y pueden atribuirse a los desechos mineros que contaminan el medio ambiente (Chirinos-Peinado y Castro-Bedriñana, 2020).

Al comparar datos de estudios realizados en otros países, los resultados siguen variando dependiendo de la localidad. Por ejemplo, en la leche cruda de nueve regiones de Corea, las concentraciones de $\mathrm{Cd}$ y $\mathrm{Pb}$ no excedieron los límites establecidos por las normas coreanas (Kim y col., 2016). En un estudio realizado en Asturias, España, se analizó la leche cruda de 7 granjas lecheras (36 muestras en total), y solo se detectó arsénico en 4 muestras con una media de $0,01845 \pm 0,00689 \mathrm{mg} / \mathrm{kg}$, y no se encontró $\mathrm{Hg}$ en ninguna muestra (González-Montaña y col., 2019). En la leche de seis granjas de cabras en Indonesia, el $\mathrm{Pb}$ se encontró a niveles entre 50 y 80 $\mathrm{mg} / \mathrm{kg}$, mientras que los niveles de As estuvieron entre 70 y $110 \mathrm{mg} / \mathrm{kg}$ (Wanniatie y col., 2019). En Turquía, el $70 \%$ de las muestras analizadas de leche 
cruda tenían $\mathrm{Pb}$ a niveles 2,5 veces superiores a los permitidos por las normas europeas. Además, el $100 \%$ de las muestras presentaron contaminación a niveles superiores al máximo permitido (Koyuncu y Alwazeer, 2019).

En otro estudio llevado a cabo en Turquía, se analizaron 90 muestras de leche y no se pudo demostrar ningún nivel detectable de $\mathrm{Pb}$. Sin embargo, As se detectó en el 94,45\% de las muestras, aunque a niveles inferiores a los estándares establecidos (Totan y Filazi, 2020). En un estudio en Rumania se trató de evaluar el riesgo de transmisión de metales pesados del forraje a la leche, pero no se evidenció riesgo alguno para la salud pública (Miclean y col., 2019). Tanto $\mathrm{Hg}$ como As se encontraron en la leche cruda en Arak, Irán, pero a niveles inferiores a las normas sugeridas por el Codex Alimentarius. En ese estudio, la concentración media de $\mathrm{Hg}$ fue significativamente mayor $(p<0,05)$ en la leche producida en las granjas tradicionales en comparación con la leche producida en las granjas industriales (Arianejad y col., 2015). Tanto Hg como As tienen el potencial de ser cancerígenos y pueden acumularse mucho más incluso a niveles inferiores a los máximos establecidos por las normativas. Sobre la base de los datos aquí presentados, la contaminación por metales pesados sigue siendo una posibilidad y se recomienda un seguimiento continuo para proteger la salud pública. Con el fin de ahondar más en la posible contaminación en el área estudiada, las futuras investigaciones deben analizar las posibles fuentes de estos metales pesados, como el agua potable, el agua de riego, los forrajes y los alimentos consumidos por las vacas lecheras en el área.

\section{Conclusiones}

El 100\% de las muestras de leche cruda analizadas en la presente investigación resultaron positivas pa-

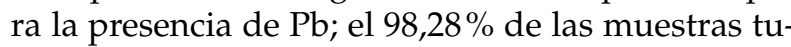
vieron niveles de $\mathrm{Pb}$ superiores al límite máximo establecido por NTE INEN 9 y el Codex Alimentarius $(0,02 \mathrm{mg} / \mathrm{kg})$. En cuanto a $\mathrm{Hg}$ y As, el 6,9\% y el 3,44\% de las muestras contenían este metal pesado, respectivamente, y a pesar que la gran mayoría de las muestras analizadas no contenían $\mathrm{Hg}$ o AS, o solo mostraron bajos niveles de estos metales pesados, estos datos siguen siendo muy preocupantes. Sobre la base de la información presentada, la conta- minación por metales pesados sigue siendo una posibilidad y se recomienda un seguimiento continuo para proteger la salud pública. Las futuras investigaciones deben estar dirigidas a investigar posibles fuentes de estos metales pesados, como el agua potable, el agua de riego, los forrajes y el alimento que reciben las vacas lecheras en el área.

\section{Agradecimientos}

Los autores agradecen a la Facultad de Medicina Veterinaria y Zootecnia y al Laboratorio DETEC de la Facultad de Ingeniería Química de la Universidad Central del Ecuador. De igual forma, a la Comisión de Investigación Formativa de la Dirección General de Investigación y Estudios de Postgrado de la Universidad Central del Ecuador por el apoyo recibido en el marco del proyecto semilla fase I. A Nydia Rodríguez, Paola Andrade y Diana Badillo por su gran colaboración. También a Michael James Stablein de la Universidad de Illinois Urbana-Champaign por sus servicios de traducción al idioma inglés y revisión de este trabajo.

\section{Referencias}

Alloway, B (2013). «Heavy metals in soils». En: Heavy metals in soils. Ed. por J. Alloway. Third Edit. Dordrecht, Springer Netherlands. Cap. Environmental Pollution.

Anastasio, A. y col. (2006). «Heavy metal concentrations in dairy products from sheep milk collected in two regions of southern Italy». En: Acta Veterinaria Scandinavica 47.1, 1-6. Online:https: / / bit.ly/2NJeA1x.

Arianejad, M. y col. (2015). «Levels of Some Heavy Metals in Raw Cow's Milk from Selected Milk Production Sites in Iran: Is There any Health Concern?» En: Health promotion perspectives 5.3, 176. Online:https://bit.ly/3onOzRV.

Ashraf, S. y col. (2019). «Phytoremediation: Environmentally sustainable way for reclamation of heavy metal polluted soils». En: Ecotoxicology and environmental safety 174, 714-727. Online:https:/ / bit.ly/3qYGOU8.

Ayala, J. y H. Romero (2013). «Presencia de metales pesados (arsénico y mercurio) en leche de vaca al sur de Ecuador». En: La Granja 17.1, 36-43. Online:https:/ / bit.ly/2NDHfF3. 
Bernhoft, R. (2012). "Mercury toxicity and treatment: a review of the literature». En: Journal of environmental and public health 2012. Online:https://bit.ly/2MqfeAh.

Bjørklund, G. y col. (2018). «Effects of arsenic toxicity beyond epigenetic modifications». En: Environmental geochemistry and health 40.3, 955-965. Online:https:/ / bit.ly/2YjGGm7.

CIL (2015). La Leche del Ecuador - Historia de la lechería ecuatoriana. Primera idición. Quito-Ecuador: Centro de la Industria Láctea.

Castro-González, N. y col. (2018a). «Heavy metals in cow's milk and cheese produced in areas irrigated with waste water in Puebla, Mexico». En: Food Additives \& Contaminants: Part B 11.1, 33-36. Online:https:/ / bit.ly/3a6Lcda.

Castro-González, N. y col. (2018b). «Metales pesados en leche de vacas alimentadas con alfalfa producida en suelos irrigados con aguas residuales en Puebla y Tlaxcala, México». En: Revista mexicana de ciencias pecuarias 9.3, 466-485. Online:https://bit.ly/3qWe6Dj.

Celis De La Rosa, A. y V. Labrada (2014). Bioestadística. Tercera Edición. Editorial El Manual Moderno.

Chirinos-Peinado, D. y J. Castro-Bedriñana (2020). «Lead and cadmium blood levels and transfer to milk in cattle reared in a mining area». En: Heliyon 6.3, e03579. Online:https:/ / bit.ly/2MfyvER.

Dergham, M. y col. (2012). «Prooxidant and proinflammatory potency of air pollution particulate matter (PM2. 5-0.3) produced in rural, urban, or industrial surroundings in human bronchial epithelial cells (BEAS-2B)». En: Chemical research in toxicology 25.4, 904-919. Online:https: / / bit . ly/36pb5nk.

GeoDatos (2019). «Coordenadas geográficas de Machachi, Pichincha, Ecuador». Online:https:/ / bit. ly/36pMbEa.

Gomes, A. y col. (2013). «Determinação de Cd, Cr e $\mathrm{Pb}$ no leite e na alimentação bovina do Brasil». En: Rev. Inst Adolfo Lutz 72.3, 211-218. Online:https://bit.ly/3csbmcX.

González-Montaña, J. (2009). «Metales pesados en carne y leche y certificación para la Unión Europea (UE)». En: Revista Colombiana de Ciencias Pecuarias 22.3, 305-310. Online:https: / / bit.ly / $36 q$ ShE9.

González-Montaña, J. y col. (2019). «Some toxic metals (Al, As, Mo, Hg) from cow's milk raised in a possibly contaminated area by different sour- ces». En: Environmental Science and Pollution Research 26.28, 28909-28918. Online:https://bit.ly/ 36pjebo.

Hashemi, M. (2018). «Heavy metal concentrations in bovine tissues (muscle, liver and kidney) and their relationship with heavy metal contents in consumed feed». En: Ecotoxicology and environmental safety 154, 263-267. Online:https: / / bit . ly/3j1HSUp.

INEC (2019). «Encuesta de Superficie y Producción Agropecuaria Continua (ESPAC) 2018». Online:https://bit.ly/3os7UBD.

INEN (2012). Leche cruda: Requisitos NTE INEN 9. Inf. téc. INEN. Online:https://bit.ly/3j7zC5C.

- (2014a). Leche y productos lácteos. Determinación del contenido de plomo. Método de espectrometría de absorción atómica en horno de grafito (ISO/TS 6733:2006, IDT). Inf. téc. INEN.

- (2014b). Leche y productos lácteos. Directrices para la toma de muestras (ISO 707:2008, IDT). Inf. téc. INEN. Online:https://bit.ly/3tbVdOC.

Karasakal, A. (2020). «Determination of trace and major elements in vegan milk and oils by ICPOES after microwave digestion». En: Biological trace element research 197.2, 683-693. Online:https: / / bit.ly/3r3UYDI.

Khairul, I. y col. (2017). "Metabolism, toxicity and anticancer activities of arsenic compounds». En: Oncotarget 8.14, 23905. Online:https: / / bit.ly / 36qUTBX.

Kim, D. y col. (2016). "Cadmium and lead in animal tissue (muscle, liver and kidney), cow milk and dairy products in Korea». En: Food Additives $\mathcal{E}$ Contaminants: Part B 9.1, 33-37. Online:https: //bit.ly/3r1noy0.

Koyuncu, Mu. y Du. Alwazeer (2019). «Determination of trace elements, heavy metals, and antimony in polyethylene terephthalate-bottled local raw cow milk of Iğdır region in Turkey». En: Environmental monitoring and assessment 191.11, 1-10. Online:https://bit.ly/3cmZw3O.

Litter, M., S. Farías y M. Armienta (2009). Metodologías Analíticas para la Determinación y Espe-ciación de Arsénico en Aguas y Suelos. Iberoarsen. Online: https:/ / bit.ly/2MGJl6s.

Miclean, M. y col. (2019). «Metal (Pb, Cu, Cd, and $\mathrm{Zn}$ ) transfer along food chain and health risk assessment through raw milk consumption from free-range cows». En: International journal of environmental research and public health 16.21, 4064. Online:https:/ / bit.ly/3cAYNfJ. 
Oliveira, T. de y col. (2017). «Direct determination of $\mathrm{Pb}$ in raw milk by graphite furnace atomic absorption spectrometry (GF AAS) with electrothermal atomization sampling from slurries». En: Food chemistry 229, 721-725. Online:https: / / bit. ly/3j07KQB.

Perrin, D., H. Schiefer y B. Blakley (1990). «Chronic copper toxicity in a dairy herd». En: The $\mathrm{Ca}$ nadian veterinary journal 31.9, 629. Online:https: //bit.ly/3ae1L6V.

Rezaeian M.and Moghadam, Ma., Mo. Kiaei y H. Zadeh (2020). "The effect of heavy metals on the nutritional value of Alfalfa: comparison of nutrients and heavy metals of Alfalfa (Medicago sativa) in industrial and non-industrial areas». En: Toxicological research 36.2, 183-193. Online:https:/ / bit.ly/36pYh02.

Rocha, E. (2011). «Espectroscopia de absorción atómica en horno de grafito y generador de hidruros». Tesis de mtría. Facultad De Ciencias Químicas UACH.

Rocha, J. y col. (2012). «Mercury toxicity». En: Journal of Biomedicine and Biotechnology, 1-2. Online:https://bit.ly/39toVai.

Rodríguez, L., E. López-Huertas y J. Boza Puerta (2010). «Tratado de Nutrición». En: ed. por Ángel Hernández. Second Edition. Madrid-España. Cap. Leche y derivados lácteos, págs. 75-106.

Samiee, F., M. Javad y M. Leili (2019). «Exposure to heavy metals released to the environment through breastfeeding: A probabilistic risk estimation». En: Science of The Total Environment 650, 3075-3083. Online:https://bit.ly/3oF2q6J.

Scaramozzino P.and Battisti, S. y col. (2019). «Application of a risk-based standardized animal biomonitoring approach to contaminated sites». En: Environmental monitoring and assessment 191.8, 1-13. Online:https://bit.ly/2NNEb9A.

Silva, C. y col. (2010). "Stable lead in milk and derivates». En: Food Analytical Methods 3.2, 85-89. Online:https://bit.ly/39wfRRP.

Totan, F. y A. Filazi (2020). «Determination of some element levels in various kinds of cow's milk processed in different ways». En: Environmen- tal monitoring and assessment 192.2, 1-10. Online:https:/ / bit.ly/2MDW4a2.

Vasconcelos, M. de y col. (2019). «Lead contamination in food consumed and produced in Brazil: Systematic review and meta-analysis». En: Food Research International 126, 108671. Online:https: / / bit.ly/3pyIqnh.

WHO (2018). «World Health Organization: Health topics of Arsenic». Online:https : / / bit . ly / $30 F c D 33$.

- (2019). «World Health Organization: Health topics of Mercury». Online:https : / / bit . ly / 3pxV6uN.

Wanniatie, V. y col. (2019). "Chemical compositions, contaminants, and residues of organic and conventional goat milk in Bogor District, Indonesia». En: Veterinary world 12.8, 1218. Online:https:/ / bit.ly/2MDSop3.

Yang, Lixin y col. (2020). «Toxicity of mercury: Molecular evidence». En: Chemosphere 245, 125586. Online:https://bit.ly/2MkCEXY.

Yilmaz, K., İ. Akinci y S. Akinci (2009). «Effect of lead accumulation on growth and mineral composition of eggplant seedlings (Solarium melongena)». En: New Zealand Journal of crop and horticultural Science 37.3, págs. 189-199.

Zhou, X. y col. (2017). "Analysis of 22 elements in milk, feed, and water of dairy cow, goat, and buffalo from different regions of China». En: Biological trace element research 176.1, 120-129. Online:https:/ / bit.ly /2L164tE.

Zhou, X. y col. (2019). «Relationships between $\mathrm{Pb}$, As, $\mathrm{Cr}$, and $\mathrm{Cd}$ in individual cows' milk and milk composition and heavy metal contents in water, silage, and soil». En: Environmental Pollution 255, 113322. Online:https : / / bit . ly / 2MkDhAO.

Zwierzchowski, G. y B. Ametaj (2018). «Minerals and heavy metals in the whole raw milk of dairy cows from different management systems and countries of origin: A meta-analytical study». En: Journal of agricultural and food chemistry 66.26, 6877-6888. Online:https://bit.ly/3j4CTT1. 